Article

\title{
Nicotinamide (aka Niacinamide) Mitigates SASP- Related Inflammation Induced by Environmental Stressors in Human Epidermal Keratinocytes
}

\author{
John C. Bierman ${ }^{1}$, Timothy Laughlin ${ }^{1}$, Makio Tamura ${ }^{1}$, Ben C. Hulette ${ }^{1}$, Catherine E. Mack ${ }^{1}$, \\ Christina Y. R. Tan ${ }^{2}$, Malgorzata Morenc ${ }^{2}$, Sophie Bellanger², and John E. Oblong1* \\ 1The Procter \& Gamble Company, Mason, OH, 45040, USA; bierman.jc@pg.com (J.C.B.); laughlin.lt@pg.com \\ (T.L); tamura.m@pg.com (T.M.) ; hulette.b.c.@pg.com (B.C.H.); mack.c.e@pg.com (C.E.M.); oblong.je@pg.com \\ (J.E.O) \\ ${ }^{2}$ Skin Research Institute of Singapore, A*STAR, Singapore; christina_tan@sris.a-star.edu.sg (C.Y.R.T.) ; \\ Malgorzata_Ewa_Morenc@sris.a-star.edu.sg (M.M.) ; sophie_bellanger@sris.a-star.edu.sg (S.B.) \\ *Correspondence: oblong.je@pg.com; Tel: +1-513-646-5064
}

\begin{abstract}
Daily exposure of skin to environmental stressors leads to molecular and morphological changes ascribed as premature aging. These stressors include solar radiation, industrial pollution, fossil fuel and carbon emissions, which cause cellular damage that induces an inflammatory response in skin. Several inflammatory components are known to be involved in triggering the senescence-associated secretory phenotype (SASP) which is known to accelerate aging. It is hypothesized that preventing induction of inflammation by environmental stressors can prevent premature aging. Since it is known that nicotinamide (Nam) has anti-inflammatory properties, we tested whether Nam can inhibit environmental stressor-induced inflammation. Exposure of keratinocytes to UVB, urban dust, diesel exhaust, and cigarette smoke extract stimulated production of the inflammatory mediators PGE2, IL-6, and IL-8 and induced gene expression patterns associated with apoptosis, DNA repair, and cell cycle control. In all cases, Nam treatment significantly inhibited these stress-induced responses. Nam also reduced IL-8 levels stimulated by the combination of topically applied particulate matter $\left(\mathrm{PM}_{2.5}\right)$ and UV exposure in 3D skin equivalents. Under $5 \% \mathrm{O}_{2}$ conditions that more closely mimic physiological $\mathrm{O}_{2}$ levels, Nam had a heightened inhibitory effect on UVB-induced PGE2 levels in keratinocytes. In a UV-challenge study, treatment with Nam reduced skin surface IL-1 $\alpha$ RA/IL-1 $\alpha$ inflammatory biomarkers and erythema that were induced by solar simulated radiation. These findings provide a body of evidence that Nam can mitigate in part the skin's inflammatory response elicited by exposure to environmental stressors. This supports the potential that Nam can inhibit premature aging and help maintain skin's functionality and appearance.
\end{abstract}

Keywords: Epidermal keratinocytes; inflammation; nicotinamide; skin; ultraviolet radiation; environmental stressors

\section{Introduction}

The skin is the largest human organ and one of its primary functions is to protect the body from exposure to damaging environmental stressors such as solar radiation, chemicals, pollution, and particulate matter. Like any organ, skin is susceptible to aging and this phenomenon can be accelerated by cumulative acute microdamage from these environmental stressors. This premature 
aging of skin leads to significant cellular and structural changes that affect skin's overall appearance, functionality, and homeostatic state. Thus, it is of interest to understand these changes in order to identify mechanistic intervention targets that would prevent premature aging and maintain skin's health and appearance.

The impact of environmental stressors on human skin has been extensively studied, particularly as it relates to solar radiation since it is accepted that ultraviolet (UV) exposure can cause upwards of $80 \%$ of premature aging [1, 2]. In addition, more recent research has studied the impact of stressors such as pollution and corresponding particulate matter on skin health [3-5]. Mechanistically one of the early events in response to environmental stressors is the generation of free radicals and reactive oxygen species (ROS) that cause DNA damage, protein structure and enzymatic activity alteration, and formation of lipid peroxides [6]. This cellular damage can be repaired via presence of antioxidant proteins and molecules as well as oxidative stress gene expression response [7]. One common feature that cascades from this initial damage is the release of inflammatory signals to trigger an innate immune infiltrate [8]. The efficiency of this response declines with age and leads to an accumulation of damage, altered gene expression patterns, and cellular and tissue changes [9]. Phenotypically, one of the hallmarks of premature aging is the presence of a chronic inflammatory state [10]. This elevated inflammation in skin tissue is hypothesized to be a major accelerator of premature aging as part of the inflamm-aging theory [11]. Thus, identifying how to reduce and prevent inflammation in response to environmental stressors is proposed as a mechanistic approach to prevent premature aging.

Nicotinamide (Nam; aka niacinamide, vitamin $\mathrm{B}_{3}$ ) has been used for decades in cosmetic and pharmaceutical products for the treatment of acne, skin photoaging attributes, and barrier integrity improvement [12-14]. It has been reported that Nam can protect cells from oxidative stress, UVinduced immunosuppression, and metabolic disruption [15-17]. Additionally, Nam has been shown to inhibit inflammatory cytokine induction by endotoxin in red blood cells [18]. Since it is not clear whether Nam can protect against other environmental stressors, we asked whether Nam can mitigate stress-induced inflammation in several skin models. Mechanistically this is of keen interested since it is known that chronic inflammation can lead to senescence and can be characterized by the senescence-associated phenotype (SASP) [19]. Components of SASP secreted by senescent cells include the cytokines IL-1 $\alpha$, IL-6, and IL-8 [20]. We had previously shown that Nam can reduce senescence induced by NAD+ metabolism modulation in keratinocytes [21]. Using telomerase immortalized keratinocytes (hTERT), we found that Nam significantly reduced the induction of the prostaglandin PGE 2 and the cytokines IL-6 and IL-8 after exposure to UVB, urban dust, diesel exhaust, and cigarette smoke extract. To more closely mimic physiological exposure conditions, we used 3D full thickness skin equivalents to treat topically with particulate matter ( $\left.\mathrm{PM}_{2.5}\right)$ in combination with ultraviolet B (UVB) exposure. Under these conditions Nam inhibited the induced IL-8 levels to the control treated levels. To more closely mimic in vivo oxygen conditions in the epidermis, we compared keratinocytes grown under $5 \%$ or $20 \% 0_{2}$ conditions [22]. Interestingly, keratinocytes grown at $5 \% \mathrm{O}_{2}$ had higher levels of basal and UV-induced PGE2 compared to cells grown at $20 \% 02$. Under both oxygen conditions, Nam significantly reduced basal and UVB-induced PGE2 levels. A double-blinded, placebo-controlled UV challenge study was conducted to test whether a $5 \%$ Nam skin care emulsion system applied to female subjects' backs could reduce inflammation from exposure to solar simulated radiation (UVA and UVB proportion of 89.5:10.5) at 1.5 minimal 
erythemal dose (MED). Treatment with Nam showed significantly lower levels of the skin surface inflammatory IL-1 $\alpha$ RA/IL-1 $\alpha$ biomarkers and overall reduction in erythema as measured by Chromameter and live visual grading in comparison to non-treated and placebo control sites.

Our results provide a body of evidence that Nam mitigates the inflammatory signal response induced by environmental stressors in several skin models. We propose that Nam can inhibit premature aging and more importantly help maintain skin's functionality and appearance. It should also be noted that while the response to Nam in these models was significant, it was partial in some instances and additional strategies in combination with Nam will be needed to further prevent the inflammatory cascade, thereby, protecting skin from cumulative damage and premature aging.

\section{Materials and Methods}

\subsection{Cells and Reagents}

Telomerase-transfected keratinocytes (hTERT) were expanded in a $\mathrm{CO}_{2}, 37^{\circ} \mathrm{C}$ incubator using Epilife $^{\mathrm{TM}}$ (ThermoFisher Scientific, Waltham, MA) media with human keratinocyte growth supplement, and gentamicin/amphotericin B. Cells never exceeded $70 \%$ confluency nor passage 5 . UVB stress was administered using a BioSun (Vilber Lourmat, Germany). Urban dust was purchased from the National Institute of Standards \& Technology (NIST), as atmospheric material collected in the Washington DC area during a 12-month period from 1976-77 (NIST 1649b). Diesel exhaust was also purchased from NIST, as a collection from a filtering-system designed for dieselpowered forklifts (NIST 2975). PM2.5 was collected from quartz tape environmentally exposed in Beijing China in 2014 and was extracted in an aqueous solvent. A cigarette smoke-extract (CSE) was prepared from burning commercial unfiltered cigarettes in a modified apparatus from a previously described set-up [23]. The process was controlled by the number of cigarettes, the length of time to burn each cigarette and the volume of collection fluid (PBS). Briefly, filters were removed from cigarettes, cigarettes were burned at a rate of 2 minutes/cigarette and smoke phase passed through 60 $\mathrm{mL}$ of PBS buffer. After collecting the smoke from 4 cigarettes, the smoke extract was removed from the capture flask and used within 2 hours of production. Treatments with stressors with and without Nam were further cultured for 20 hours before harvest.

A 3D full thickness human skin organotypic model (EpiDermFTTM, MatTek Corporation, Ashland, MA) was used to assay the effects of Nam and stressors. Briefly, epidermal/dermal cultures were received and allowed to acclimate overnight in Dulbecco's Modified Eagle's Medium (DMEM) with epidermal growth factor, insulin, hydrocortisone and proprietary stimulators of epidermal differentiation supplement, and gentamicin/amphotericin B in a humidified atmosphere with $5 \% \mathrm{CO} 2$ at $37^{\circ} \mathrm{C}$. Prior to treatments, cultures were lifted to the air-liquid interface as per manufacturer's instructions. After treatment, media was collected and analysed for IL-8. Cultures were manually separated into epidermal and dermal fractions and subjected to microarray profiling on the GeneTitan U219 array platform (Affymetrix, Santa Clara, CA) as previously described [21].

\subsection{ATP Measurement}

ATP was quantified from cells in culture by CellTiter-Glo® assay (Promega, Madison, WI). The media was removed, cells were washed with $200 \mu \mathrm{L}$ of PBS, and $100 \mu \mathrm{L}$ of media was added to each well. CellTiter-Glo reagent was prepared according to manufacturer's instructions and $100 \mu \mathrm{L}$ were 
added to cells. Cells were incubated with reagent per manufacturer's instructions and $200 \mu \mathrm{L}$ were transferred from the 24 well V7 plate to a black clear bottom 96 well plate in order to quantify ATP via luminescence. A minimum of 3 separate experiments were performed and data were analyzed from either 3 replicates. Student's t-test was used for statistical analysis with data presented as average value and standard deviation.

\subsection{PGE2, IL-6 and IL-8 Measurements}

For PGE2 experiments, the media was supplemented with $2.5 \mu \mathrm{g} / \mathrm{mL}$ arachidonic acid. PGE2 was measured from culture supernatants using an HTFR kit (CisBio, Bedford, Massachusetts) and normalized to ATP values derived from the cells. The cytokines IL-6 and IL-8 were measured using Mesoscale Discovery platform and kits.

\subsection{Lamin B1 and Ki-67 Immunofluorescence Staining}

Usage of primary human keratinocytes was approved by A*STAR Institutional Review Board (A*STAR IRB reference: 2019-53) and the National University of Singapore (NUS) Institutional Review Board (NUS-IRB reference: B-14-257E).

Human Primary Keratinocytes (HPKs) were obtained from healthy human skin samples (6-year old male donor, foreskin) from de-identified surplus surgical waste with written informed patient consent and ethical clearance. HPKs were isolated as previously described [22]. For maintenance, HPKs were cultured on lethally irradiated murine 3T3-J2 feeder cells in cFAD medium (3:1 DMEM/Ham's F-12) supplemented with 10\% fetal calf serum (FCS), $1 \%$ penicillin/streptomycin and $10 \mathrm{ng} / \mathrm{ml}$ epidermal growth factor as previously described [22]. The medium was replaced every 23 days.

One day before irradiation, HPKs were seeded in a feeder-free system in Dermalife ${ }^{\circ}$ medium (LL-0007; Lifeline Cell Technology, Oceanside, CA). Medium was changed just before irradiation (the volume was reduced by 5 times for irradiation) and cells were irradiated with $25 \mathrm{~mJ} / \mathrm{cm}^{2} \mathrm{UVB}$ using a BioSun (Vilber-Lourmart, Germany). The medium was changed again immediately after irradiation and cells were maintained for $72 \mathrm{~h}$ in medium with or without $1.5 \mathrm{mM}$ Nam (for Nam-treated cells, $1.5 \mathrm{mM}$ Nam was also added to the medium just before irradiation).

Seventy-two hours after irradiation, cells were fixed in $2 \%$ paraformaldehyde at $4{ }^{\circ} \mathrm{C}$ for 30 minutes, permeabilized with PBS/0.1\% Triton, blocked with $\mathrm{PBS} / 10 \%$ goat serum, incubated with primary antibodies (Lamin B1 (1/800): Novus NBP2-48966; Ki-67 (1/100): DAKO M7240), washed in PBS and incubated with secondary antibodies (Alexa Fluor, ThermoFisher Scientific, Waltham, MA) and DAPI $(1 \mu \mathrm{g} / \mathrm{mL})$ before washing and mounting (Vectashield $®$; Vector Laboratories, Burlingam, CA). Images were acquired on an Olympus FLUOVIEW FV3000 RS inverted confocal microscope and quantitated in ImageJ.

\subsection{Eigene Gene Analysis}

Cells were lysed (RLT Buffer, Qiagen, Germantown, Maryland) and RNA was isolated using Agencourt magnetic beads (BeckmanCoulter, Indianapolis, IN). Targets were prepared according to manufacturer's instructions and analyzed on Affymetrix U219 GeneChips ${ }^{\circledR}$ (Affymetrix, Santa Clara, CA). Gene expression changes of Apoptosis, DNA repair, and Cell Cycle biological processes in GeneOntology (GO:0006915, GO:0006281, and GO:0007049, respectively) were assessed by using Eigenegene expression [25]. The Eigenegenes of Apoptosis, DNA repair, and Cell Cycle consisted 
of 415,243 , and 352 genes expressed in keratinocytes, respectively, and capture $\sim 30 \%$ of the variances for the changes between $0.5 \%$ CSE treatment and vehicle control.

\subsection{UV Challenge Study}

This was a four-week double-blinded placebo-controlled study in healthy female subjects, ages 18-50, with Fitzpatrick Skin Types II-III, that employed a randomized, complete block design, in which 12 treatments were evaluated on twelve $3 \times 3 \mathrm{~cm}$ sites on the subjects' backs. The study was performed between January and February 2012 at the Consumer Product Testing Company (Fairfield, NJ, USA). Good clinical practices were followed, and all subjects gave their informed consent for inclusion before they participated in the study. Due to the cosmetic nature of this study, an IRB approval was not obtained. The subjects were divided into 2 groups (one group with 31 enrolled, and one with 32 enrolled) that were staggered by 1 week. Fifty-nine subjects completed the study. There was a 1-week wash-out period, during which time each subject's minimal erythemal dose (MED) was determined for calculating the UV exposure dose to deliver on study day 16. Wash-out was followed by 2 weeks of daily test product application $\left(3 \mu \mathrm{l} / \mathrm{cm}^{2}\right)$ conducted at the study facility before a single controlled UV exposure of 1.5 MED or target $a^{*}$ value between 13-19 on each site. Daily product applications were continued on the day of UV exposure and the next 2 consecutive days. Some treatment applications occurred on Saturdays due to scheduling logistics. At evaluation visits, all measurements and imaging were completed before treatments were applied. Measurements were obtained in a controlled temperature and humidity environment $\left(70 \pm 2^{\circ} \mathrm{F}\right.$ and $30-45 \%$ R.H.). Measurements taken on each treatment site included live visual redness grading by 2 trained graders, Chromameter, and full back images at baseline, day 15 (pre-UV exposure), days 16-18 (post-UV exposure). Additionally, Chromameter measurements were taken at the time of MED determination as an exploratory measure to compare with visual grading. Four separate D-squame ${ }^{\circledR}$ tape strips were collected from the non-treated and treated sites at baseline (day 0), at end of pretreatment prior to UV exposure (day 15), and at 7, 10, and 14 days post UV exposure (days 23, 26 and 30). Total protein was extracted from the tapes and analyzed for IL-1 $\alpha$ RA and IL-1 $\alpha$ [26].

\section{Results}

\subsection{Nam Protects Cell Morphology and Mitigates UV-Induced Inflammatory Response}

Human hTERT keratinocyte cultures were used to determine the impact of Nam on mitigating UVB-induced inflammatory response. Cell morphology and viability were evaluated along with quantitation of PGE2 levels following exposure to a UVB dose response range between $50-100 \mathrm{~mJ} / \mathrm{cm}^{2}$. Additionally, cultures were pretreated with and without $500 \mathrm{mM}$ Nam prior to UVB exposure (Fig 1). Bright field images along with cell viability showed that Nam had a detectable protective effect at $75 \mathrm{~mJ} / \mathrm{cm}^{2}$ UVB. Treatment with Nam significantly reduced PGE2 levels induced at 50 (32\%), 75 (22\%), and $100(40 \%) \mathrm{mJ} / \mathrm{cm}^{2} \mathrm{UVB}$. 


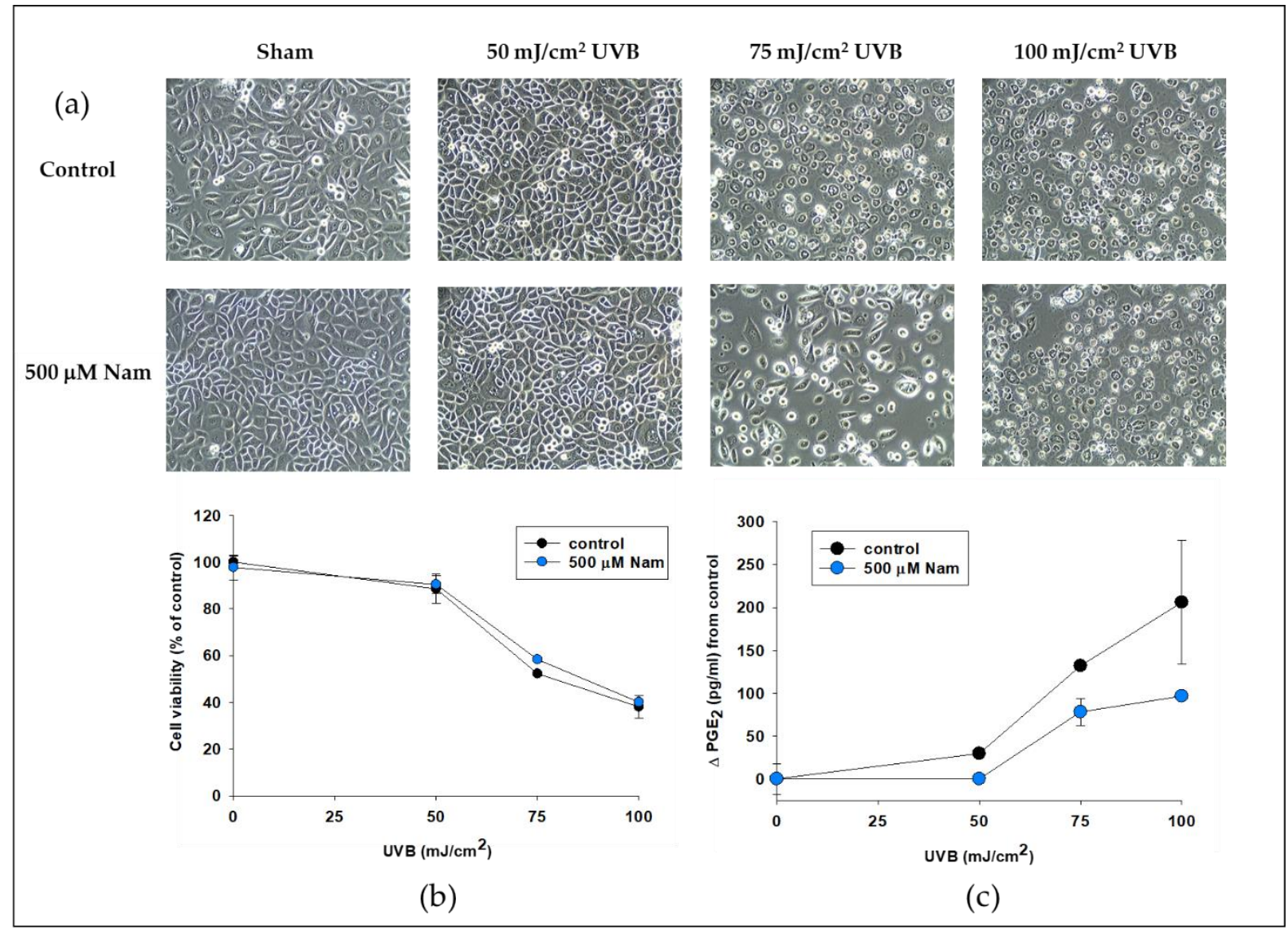

Figure 1. Nam partially protected keratinocyte viability and reduced PGE2 levels induced by UVB exposure. Keratinocytes were incubated for 24 hours with or without $500 \mu \mathrm{M} \mathrm{Nam}$ and exposed to 50,75 and $100 \mathrm{~mJ} / \mathrm{cm}^{2}$ of UVB after media was changed to PBS. Keratinocytes were then incubated for 24 hours in full media with or without $500 \mu \mathrm{M}$ Nam. (a) Bright field image capture across treatment groups shows significant cell morphology changes with increasing UVB fluency. Treatment with $500 \mu \mathrm{M}$ Nam partially protected cell morphology changes from $75 \mathrm{~mJ} / \mathrm{cm}^{2}$ UVB. (b) Cell viability (ATP quantitation) was lower across all fluencies and there was a statistically significant mitigation by Nam at $75 \mathrm{~mJ} / \mathrm{cm}^{2} \mathrm{UVB}$ exposure. (c) Quantitation of inflammatory response showed a significant reduction in secreted PGE2 levels by $500 \mu \mathrm{M}$ Nam at all UVB fluencies tested. A replicate of $n=4$ were averaged and Student's t-test was performed to calculate statistical significance $\left({ }^{*} p<0.05\right.$, $\left.{ }^{* *} \mathrm{p}<0.01\right)$.

We then tested whether Nam can impact the inflammatory response post UVB exposure as well as measured additional inflammatory signaling agents. hTERT keratinocytes were exposed to 50 $\mathrm{mJ} / \mathrm{cm}^{2}$ of UVB and incubated overnight with and without $500 \mu \mathrm{M}$ Nam. Nam treatment after UVB exposure showed an increase in UVB-induced PGE2 as well as the SASP-associated inflammatory cytokines IL-6 and IL-8. Treatment with Nam resulted in a 30\% reduction in PGE2 levels, 31\% reduction in IL-6 levels, and 21\% reduction of IL-8 levels (Fig 2a). Since SASP induction correlates with an onset of senescence, we measured changes in $\mathrm{Ki}-67$ as an indicator of proliferation and Lamin B1 (LmnB1), a nuclear scaffold protein known to be lost during the senescence process [27]. To better simulate senescence response, we utilized primary human keratinocytes in place of the immortalized hTERT keratinocytes. We had found that UVB exposure of primary keratinocytes causes a similar increased PGE2 that is inhibited by Nam exposure. UVB treatment at $25 \mathrm{~mJ} / \mathrm{cm}^{2}$ induced a dramatic 
increase in cell size and a significant loss of both LmnB1 and Ki-67, all indicative of senescence (Fig $2 b$ ). A substantial number of cells (shown by yellow arrows) expressed levels of LmnB1 barely detectable by immunofluorescence 72 hours after UVB irradiation. Interestingly, addition of Nam partially prevented the drop of LmnB (although most cells still appeared larger than control cells and the confluency was lower than in the control population). Accordingly, proliferation was partially preserved by Nam as shown by maintenance of numerous Ki-67-positive cells as compared to UVB alone. Of note, all Ki-67-positive cells were strongly positive for LmnB1 as expected (consistently, cells negative for Ki-67 expressed no or very low levels of LmnB1). Quantitation of immunofluorescent images confirmed the significant decrease in LaminB1 with UVB exposure and restoration with Nam treatment (Fig 2c).

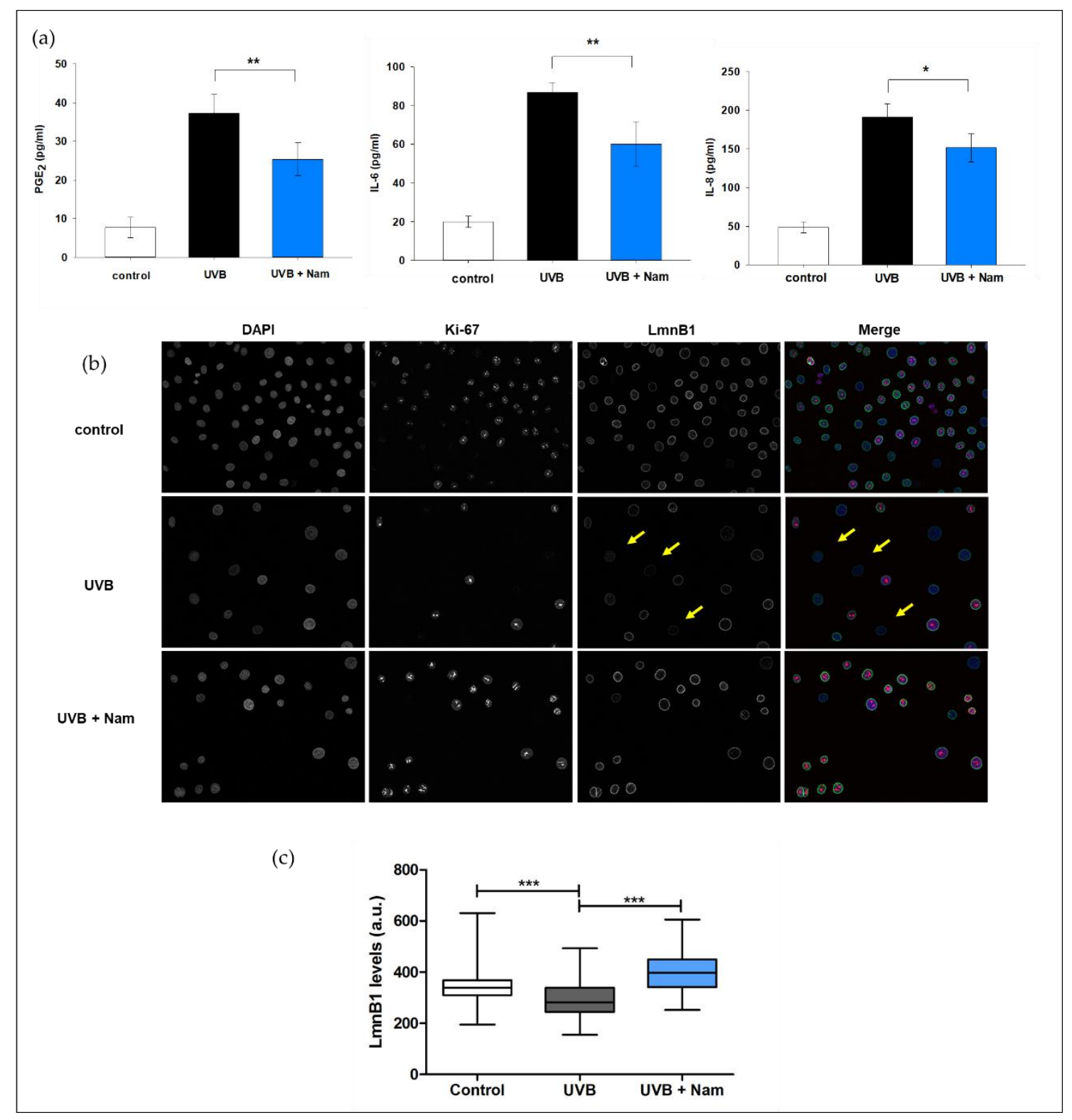

Figure 2. Nam reduced synthesis of inflammatory signals and restored Lamin B1 levels in keratinocytes after exposure to UVB. (a) hTERT keratinocytes were cultured for two days in full media and switched to PBS prior to being exposed to $50 \mathrm{~mJ} / \mathrm{cm}^{2}$ of UVB radiation. After irradiation, $500 \mu \mathrm{M}$ Nam was added to the culture wells in full media, incubated for 24 hours, and PGE2, IL-6, and IL-8 media levels quantitated. Nam treated cells 
showed a significant decrease in levels of PGE2, IL-6, and IL-8. A replicate of $n=4$ were averaged and Student's t-test was performed to calculate statistical significance $\left({ }^{*} \mathrm{p}<0.05,{ }^{* *} \mathrm{p}<0.01,{ }^{* * *} \mathrm{p}<0.001\right)$. (b) Immunofluorescence staining for the senescence biomarker Lamin B1 (LmnB1) showing protective effect of $1.5 \mathrm{mM}$ Nam on human primary keratinocytes exposed to $25 \mathrm{~mJ} / \mathrm{cm}^{2} \mathrm{UVB}$. Nam treatment also partially protected against loss of cell proliferation triggered by UVB as measured by Ki-67 and DAPI staining. (c) Quantitation of LmnB1 immunofluorescence images showed that $25 \mathrm{~mJ} / \mathrm{cm}^{2}$ of UVB significantly decreased LmnB1 levels while treatment with Nam significantly prevents the LmnB1 drop. GraphPad Prism Version 5.03 was used for statistical analyses. A one-way ANOVA test ( $\left.{ }^{* * *} \mathrm{p}<0.001\right)$ was applied, whiskers represent Min to Max values, $\mathrm{n}>150$ cells per condition.

\subsection{Secretion of Inflammatory Signals and Gene Expression Patterns Induced by Environmental Stressors in Keratinocytes Is Mitigated by Nam}

In addition to UVB, we tested whether other environmental stressors can induce SASP-related inflammation in hTERT keratinocytes at non-cytotoxic levels. hTERT keratinocytes were exposed to $0.00028 \%$ diesel exhaust and $0.0075 \%$ urban dust in media with and without $500 \mu \mathrm{M}$ Nam. After 24 hours of exposure, IL-8 levels were quantitated from culture media (Fig 3a). Compared to basal levels, both diesel exhaust and urban dust stimulated a 39\% and 100\% increase in IL- 8 levels, respectively. Nam treatment showed a complete inhibition of IL-8 induction by diesel exhaust and a $21 \%$ reduction in levels induced by urban dust. A cigarette smoke extract (CSE) was made using cigarette smoke percolated through a water capture system [23]. Exposure to 0.05\% CSE showed a 95\% increase in PGE2 levels and treatment with $500 \mu \mathrm{M}$ Nam caused a complete rescue to basal levels (Fig 3b).

Additionally, hTERT keratinocytes were exposed to CSE with and without $500 \mathrm{mM}$ Nam, incubated overnight and then processed for mRNA isolation and microarray analysis. Transcriptomics profiling of key bio-themes showed that CSE induced gene expression patterns associated with apoptosis, DNA repair, and cell cycle regulation in a dose-dependent manner (Fig 3c). Interestingly Nam treatment showed an ability to partially reverse the CSE-induced changes. 


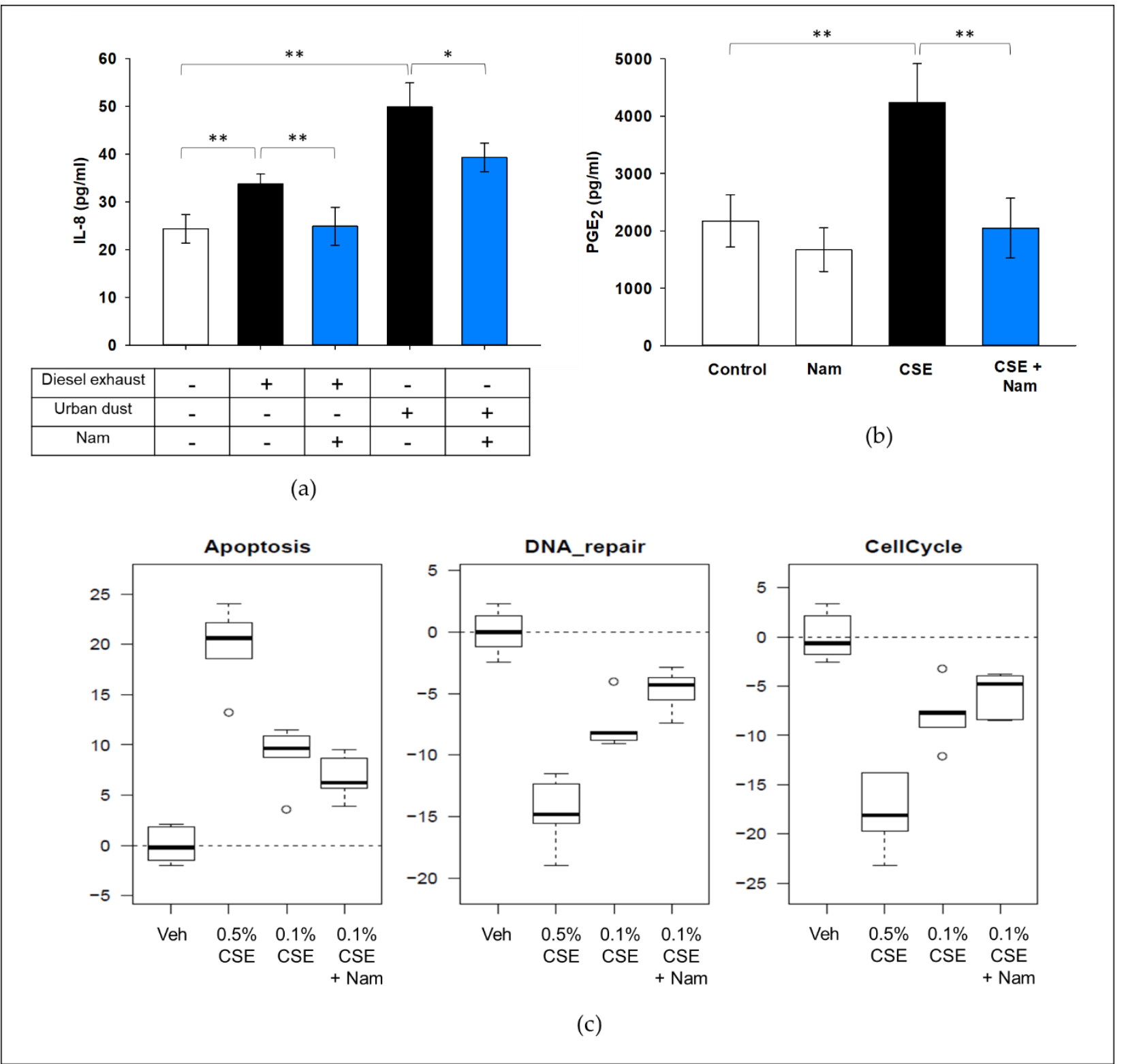

Figure 3. Nam reduced inflammatory mediator levels induced by environmental stressors in keratinocytes. hTERT keratinocytes were incubated in full media for 24 hours and then exposed to diesel exhaust, urban dust or cigarette smoke extract (CSE) with or without $500 \mu \mathrm{M}$ Nam for 24 hours. IL-8 and PGE2 levels in media were quantitated via ELISA. (a) $500 \mu \mathrm{M}$ Nam co-treatment limited induction of IL-8 levels by both diesel exhaust and urban dust. (b) $500 \mu \mathrm{M}$ Nam prevented induction of PGE2 by $0.5 \%$ cigarette smoke extract (CSE). A replicate of $n=4$ were averaged and Student's t-test was performed to calculate statistical significance $\left({ }^{*} \mathrm{p}<0.05\right.$, ${ }^{* *} \mathrm{p}<0.01$ ). (c) Transcriptomics profiling of changes in top bio-themes in hTERT keratinocytes following exposure to $0.1 \%$ and $0.5 \%$ CSE showed a significant increase in apoptosis and decreases in DNA repair and cell cycle related genes in a dose-dependent manner. Concurrent treatment with $500 \mu \mathrm{M}$ Nam and 0.1\% CSE showed that Nam was able to partially prevent the degree of response to CSE in keratinocytes.

\subsection{Nam Reverses Inflammatory Signal Response Induced by UV and PM 2.5 Combination in 3D}

\section{Skin Equivalent Models}

Particulate matter ( $\left.\mathrm{PM}_{2.5}\right)$ is comprised of carbon-based matter that ranges in particulate size and is bound with various chemical agents [28]. While the chemical agents can be extracted and tested, 
we wished to more closely simulate physiological exposure of $\mathrm{PM}_{2.5}$ that comes in direct contact with skin. Additionally, we tested the combination effect of PM2.5 with UV exposure by using an equivalent to solar radiation of UVA/UVB at a respective proportion of 89.5:10.5. 3D full thickness skin equivalent cultures (EpiDermFT ${ }^{\mathrm{TM}}$, MatTek Corporation, Ashland, MA) were stressed with topically applied $\mathrm{PM}_{2.5}$ and with and without exposure to $250 \mathrm{~mJ} / \mathrm{cm}^{2}$ of $\mathrm{UV}$. Cultures were also treated with $500 \mu \mathrm{M}$ Nam in full media and incubated overnight (24 hours) prior to stress and immediately following stress. PM 2.5 and UVB exposures alone caused a $40 \%$ and $21 \%$, respectively, increase in secreted IL-8 levels whereas the combination led to a synergistic increase of $85 \%$ compared to basal levels (Fig 4). Treatment with Nam caused a complete inhibition of IL-8 induction stimulated by the combination of $\mathrm{PM}_{2.5}$ and UV.

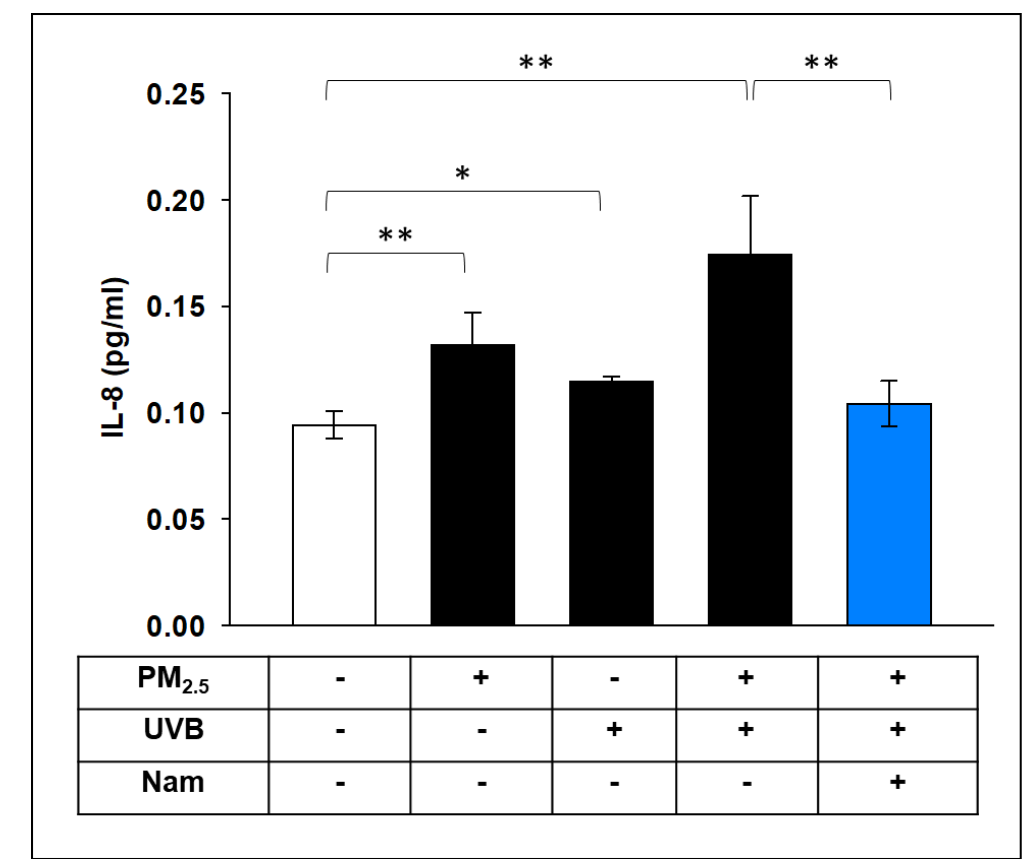

Figure 4. Nam limited IL-8 induction by particulate matter alone and in combination with UV in 3D skin equivalents. EpiDermFTTM 3D skin equivalent cultures were acclimated for 24 hours in full media and then exposed to topical particulate matter $\left(\mathrm{PM}_{2.5} ; 0.12 \mathrm{ug} / \mathrm{ml}\right)$ alone and in combination with $250 \mathrm{~mJ} / \mathrm{cm}^{2} \mathrm{UVA} / \mathrm{UVB}$ (89.5:10.5). $500 \mu \mathrm{M}$ Nam was added to the media of select treatment groups and incubated for 24 hours prior to stress and immediately following stress. IL-8 levels were quantitated via ELISA. The combination of UV and PM2.5 additively induced IL-8, while Nam treatment significantly prevented IL-8 induction. A replicate of $\mathrm{n}=4$ were averaged and Student's t-test was performed to calculate statistical significance $\left({ }^{*} \mathrm{p}<0.05,{ }^{* *} \mathrm{p}<0.01\right)$.

\subsection{Heightened Inhibition of UV-Induced PGE 2 Synthesis by Nam Under $5 \% \mathrm{O}_{2}$ Culture Conditions}

It has been reported that $0_{2}$ levels measured in human epidermis can range between 0.2 and $8 \%$ while exceeding $7 \%$ in dermis [23]. Thus, we wished to understand whether keratinocytes grown under conditions that more closely mimic physiological oxygen levels have any differential response to stress and Nam. To test this, hTERT keratinocytes were grown for 48 hours at $20 \%$ and $5 \% 0_{2}$ culture conditions. After acclimation, cells were exposed to $25 \mathrm{~mJ} / \mathrm{cm}^{2} \mathrm{UVB}$, treated with $500 \mu \mathrm{M}$ Nam, and incubated overnight under similar oxygen conditions. Interestingly, control keratinocytes grown at $5 \% \mathrm{O}_{2}$ showed a $200 \%$ increase of $\mathrm{PGE}_{2}$ compared to cells grown at $20 \%$ (Fig 
5). UVB exposure of cells grown at $5 \% 0_{2}$ induced a $167 \%$ increase of $\mathrm{PGE}_{2}$ compared with a $78 \%$ increase for cells grown at $20 \% 0_{2}$. This suggests that keratinocytes can show a dynamic and heightened inflammatory response range under more normoxic conditions. Treatment with Nam showed similar responses between the two oxygen conditions but a heightened inhibitory effect at $5 \%$ 02. Nam reduced control $\mathrm{PGE}_{2}$ levels by $50 \%$ in cells grown at $5 \% \mathrm{O}_{2}$ compared with $23 \%$ reduction at $20 \% 0_{2}$. After UVB exposure, Nam reduced $\mathrm{PGE}_{2}$ levels by $57 \%$ at $5 \% \mathrm{O}_{2}$ in contrast to $22 \%$ in cells grown at $20 \% \mathrm{O}_{2}$. These results demonstrate that the overall responsiveness of keratinocytes to UVB on PGE2 induction as well as reduction by Nam are similar under either $5 \%$ or $20 \% 0_{2}$ conditions. However, the stronger effect of Nam in more normoxic conditions suggests a greater potential for in vivo impact.

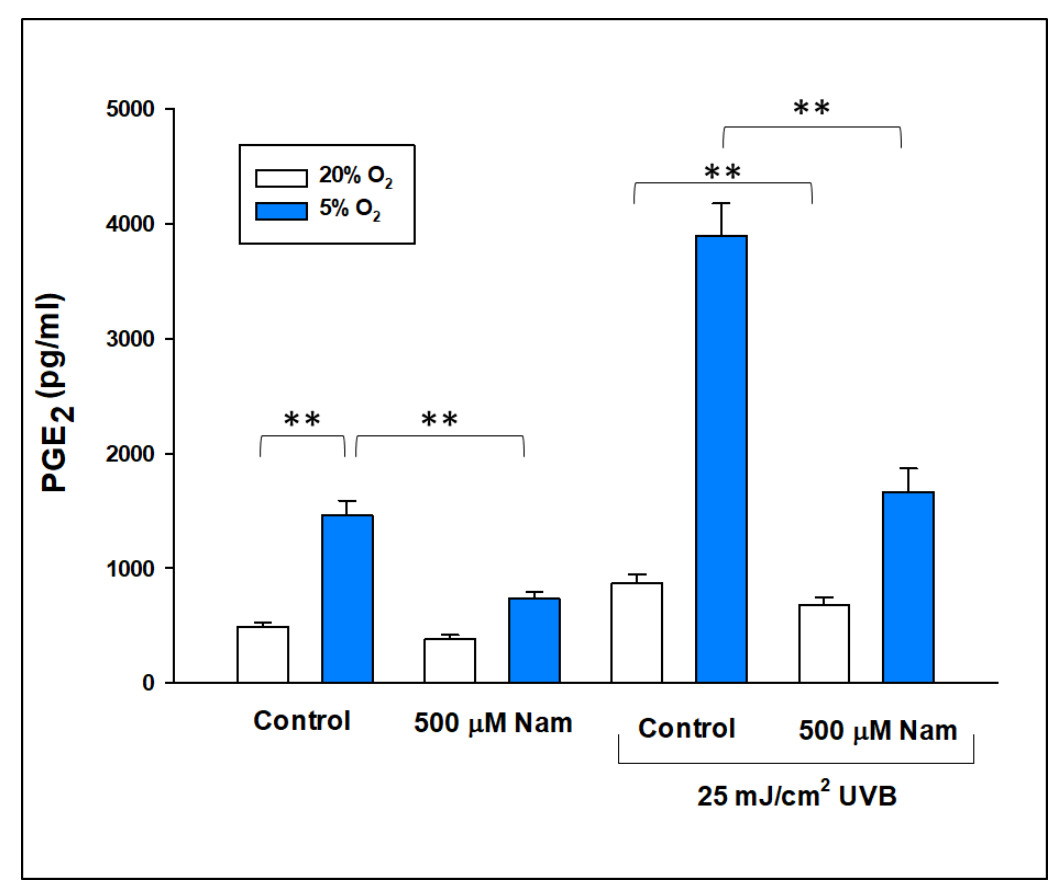

Figure 5. Nam inhibited a heightened inflammatory response to UVB in keratinocytes cultured at $5 \% 02$. hTERT keratinocytes were incubated in full media for two days under $20 \%$ or $5 \% 0_{2}$ conditions. Cultures were first exposed to $25 \mathrm{~mJ} / \mathrm{cm}^{2} \mathrm{UVB}$ and then incubated with or without $500 \mu \mathrm{M}$ Nam for 24 hours. PGE 2 levels in culture media were quantitated via ELISA. Basal levels of PGE2 synthesized from control keratinocytes were significantly higher under $5 \% \mathrm{O}_{2}$ and showed a significantly higher increase after UVB exposure compared to $20 \%$ 02. Nam treatment significantly reduced PGE2 levels at all oxygen and UVB exposure conditions. The maximal percentage inhibition by Nam was obtained at $5 \% 0_{2}$ after UVB exposure. A replicate of $n=4$ were averaged and Student's t-test was performed to calculate statistical significance $\left({ }^{* *} \mathrm{p}<0.01\right)$.

\subsection{Treatment with Topical 5\% Nam Can Reduce UV-Induced Erythema and the Ratio of the Skin} Surface Inflammatory Biomarkers IL-1 $\alpha$ RA/IL-1 $\alpha$

Based on our in vitro data, we wished to test whether Nam could impact UV-induced inflammation in skin. A double-blinded, placebo-controlled UV challenge study was designed with controlled on-site daily application (minus weekends) of an oil-in-water emulsion placebo vehicle control, 5\% Nam, and a no treatment site. After 2 weeks of product application, all sites were exposed to a single 1.5 MED dose of solar simulated radiation (UVA and UVB proportion of 89.5:10.5). 
After exposure, panelists continued to have product applied for an additional 2 weeks. Clinical measures were collected at varying time points before and after UV exposure.

To measure the effect of $5 \%$ Nam treatment on UV-induced inflammatory response, D-squame® tape strips were used to collect skin surface material for quantitation of the inflammatory biomarkers IL-1 $\alpha$ RA and IL-1 $\alpha$. The ratio of these cytokines has been previously reported to provide a measure of the underlying inflammatory state of skin in such conditions as UV-induced sun damage, dandruff, and diaper dermatitis [29]. Comparison across the various sites and time points showed a significant increase in the relative ratio of IL-1 $\alpha$ RA/IL-1 $\alpha$ after UV exposure (Fig 6a). Comparing not treated and vehicle treated sites with $5 \%$ Nam, treated sites showed a significant reduction of the IL- $1 \alpha$ RA/IL- $1 \alpha$ ratio at all time points after UV exposure (Fig 6a, arrow). At 14 days after UV exposure, $5 \%$ Nam had a maximal inhibitory effect of $18 \%$ and $16 \%$ compared to non-treated $(\mathrm{p}=0.0046)$ and vehicle treated $(\mathrm{p}=0.008)$ sites, respectively (Fig 6a, day 30). Additionally, overall analysis showed a $16 \%$ reduction by $5 \%$ Nam compared to vehicle control ( $p=0.001$ ) (Fig 6b). These data support the erythema measures where Treatment with $5 \%$ Nam can partially mitigate the inflammatory response induced by UV. Interestingly, while there were no significant differences between non-treated sites and vehicle control at individual time points, overall analysis showed vehicle control sites had a significantly lower level of the inflammatory ratio $(p=0.012)$.

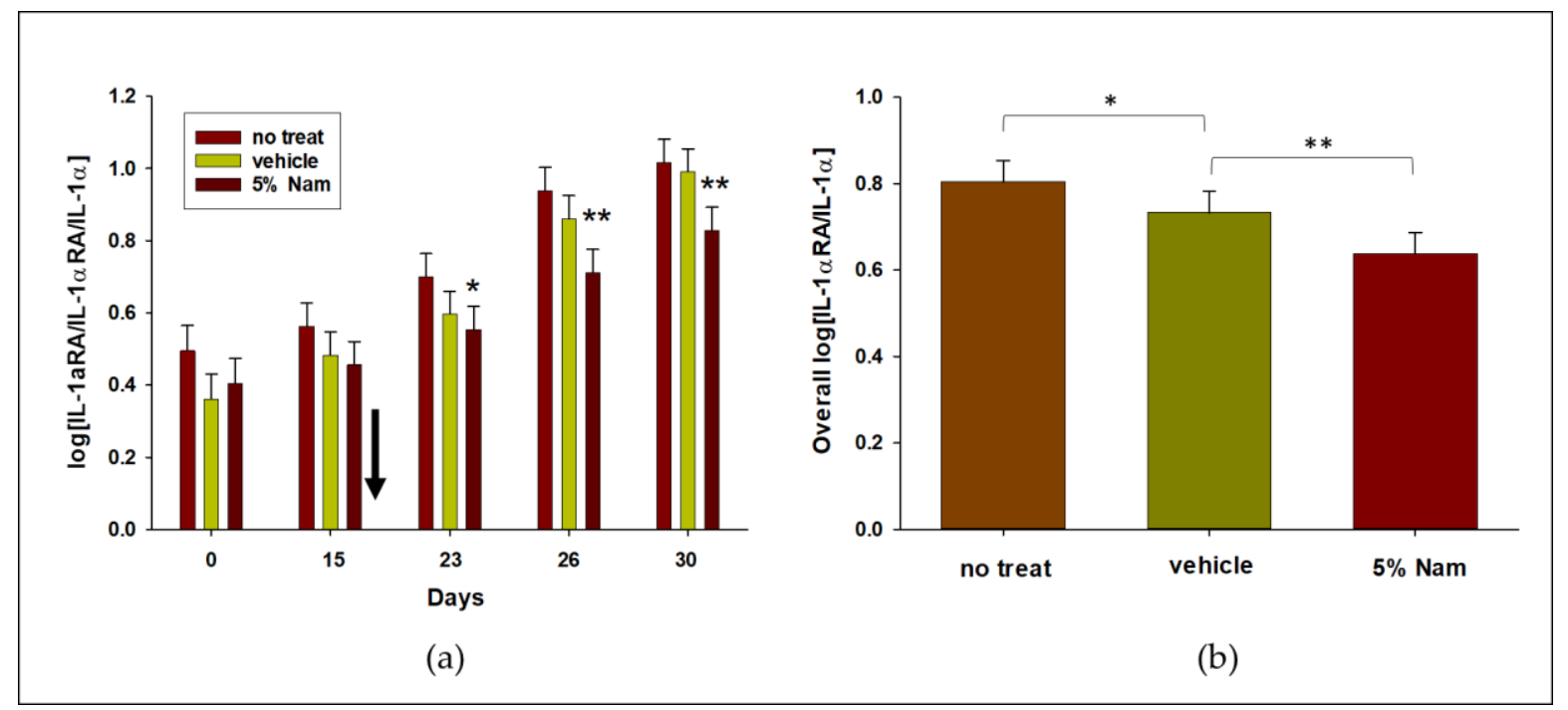

Figure 6. Treatment with topical Nam reduced the ratio of the skin surface inflammatory biomarkers IL-1 $1 \alpha \mathrm{RA} / \mathrm{IL}-1 \alpha$. Female panelists were treated on a $3 \mathrm{~cm}$ x $3 \mathrm{~cm}$ site on their backs for 14 days with a skin care emulsion vehicle with or without $5 \%$ Nam. At day 16, the treatment sites, including a non-treated site (no treat) were exposed to solar simulated radiation (UVA and UVB proportion of 89.5:10.5) to induce a 1.5 minimal erythema dose (MED). Four separate Dsquame ${ }^{\circledR}$ tape strips were collected from each site at baseline (day 0), after pretreatment phase (day 15) and 7, 10, and 14 days after UV exposure (days 23, 26, and 30). (a) Quantitation of extracted IL-1 $\alpha$ RA/IL-1 $\alpha$ protein ratio showed a significant reduction in 5\% Nam versus no treat at day 7 post UV exposure (day 23, p=0.010) and versus vehicle control at days 10 and 14 post UV exposure (day 26, $\mathrm{p}=0.008$; day 30, $\mathrm{p}=0.005$ ). (b) Overall IL-1 $\alpha \mathrm{RA} / \mathrm{IL}-1 \alpha$ protein ratios showed that $5 \%$ Nam induced a ratio significantly reduced compared to vehicle control $(\mathrm{p}=0.001)$, while vehicle control also showed a ratio reduced compared to non-treated sites although less significant (no treat, $\mathrm{p}=0.012$ ). 
Relative to UV-induced erythema, 5\% Nam showed a numerically directional effect in reducing chromameter $a^{*}$ values compared to no treatment and vehicle control sites (Fig 7a, b). Visual grading for redness showed a similar effect of Nam compared to vehicle sites (Fig 7c). Digital images captured two days after UV exposure further confirmed the ability of $5 \%$ Nam to partially prevent UV-induced redness (Fig 7d). There was no significant difference between no treat and vehicle control sites (data not shown).

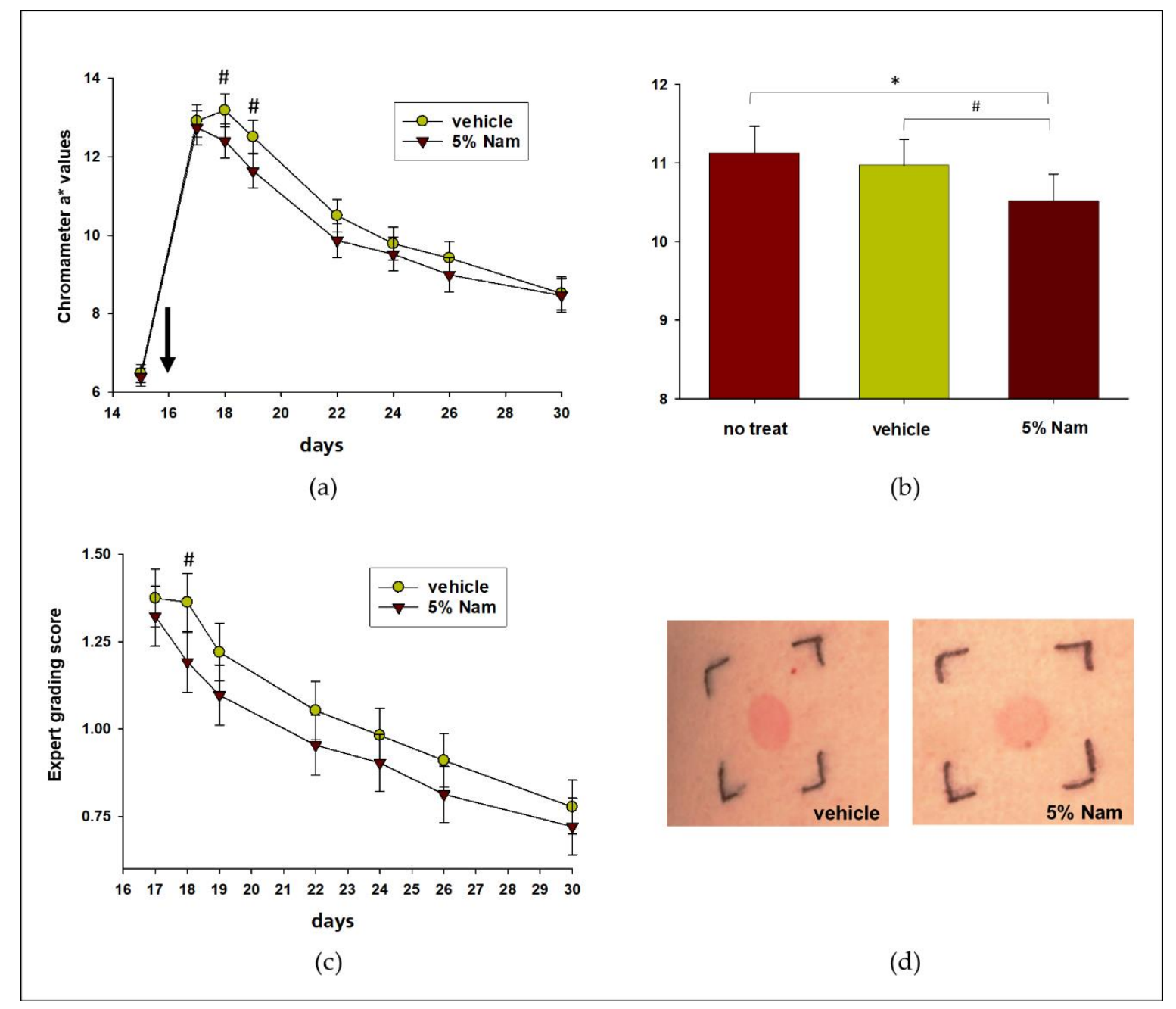

Figure 7. Treatment with Nam reduced erythema induced by solar simulated radiation. Female panelists were treated on a $3 \mathrm{~cm} \times 3 \mathrm{~cm}$ site on their backs for 14 days with an emulsion vehicle control and an emulsion containing 5\% Nam. At day 16, the treatment sites, including a non-treated site (no treat) were exposed to a 1.5 minimal erythema dose (MED) with solar simulated radiation (UVA and UVB proportion of 89.5:10.5). Chromameter $\mathrm{a}^{*}$ value readings were collected at the end of the pretreatment phase (day 15) and at 7 separate visits post UV exposure (arrow denotes day 16 of MED exposure). Treatment with $5 \%$ Nam showed directional reduction in $\mathrm{a}^{*}$ values compared to vehicle control 2 and 3 days after UV exposure (day 18, \#p=0.085; day 19, $\# p=0.057)$. (b) Overall Chromameter $a^{*}$ values calculated post UV exposure showed a significant reduction by the $5 \%$ Nam treatment compared to the non-treated sites (no treat, ${ }^{*} \mathrm{p}=0.027$ ) and vehicle control $(\# \mathrm{p}=0.083$ ). (c) Visual grading for erythema utilized a 6-point redness scale to calculate redness between treatment groups during resolution phase. Treatment with $5 \%$ Nam resulted in an overall redness profile that was directionally lower than vehicle control 2 days after UV exposure (day 18, $\mathrm{p}=0.065$ ). (d) A representative set of digital color images comparing vehicle control and 5\% Nam treatment 2 days after UV exposure (day 18). 


\section{Discussion}

Cumulative exposure of human skin to environmental stressors is one of the biggest factors that leads to molecular reprogramming and structural changes associated with premature aging. It is of importance to mechanistically understand the immediate effects from an acute exposure that can serve as part of an intervention strategy to block the negative impact and prevent premature aging. Over the past 10 years there has been increasing research to better understand the impact of environmental stressors such as pollution emissions and urban residency on skin [30]. It is known that the major impact of emitted gases and particulate matter from industrial and automotive sources on human health are respiratory effects due to direct contact, but researchers suggest that these stressors can also affect secondary organs [31, 32]. This includes reactions by skin in the form of atopic dermatitis, psoriasis, and eczema flareups $[33,34]$ and evidence that these stressors can negatively impact skin aging and appearance [5]. Cigarette smoke exposure is similar in that the cardiorespiratory health risks are established and secondary effects on skin appearance have been confirmed [35]. To better understand the molecular inflammatory response to these stressors, we utilized both in vitro and in vivo models to assess the degree of acute response to controlled exposure conditions. We further investigated whether Nam, a known vitamin that has been previously shown to have antioxidant and anti-inflammatory properties, can protect against these inflammatory responses.

Similar to what is known with UV exposure, pollution (particulate matter) has been reported to trigger inflammation in keratinocytes [36,37]. While an inflammatory response can resolve poststress, it has been suggested that this resolution becomes less efficient with aging and leads to a persistent sub-chronic inflammatory state. This has been proposed as the basis for "inflamm-aging" that leads to premature aging of tissue and disease $[38,11]$. A persistent inflammatory state can also lead to senescence that is hallmarked by the senescence-associated secretory phenotype (SASP) [20]. Major components of SASP include the cytokines IL-1 $\alpha$, IL-6 and IL-8 [19]. Thus, we wished to understand the impact of environmental stressors on the release of these SASP associated cytokine biomarkers as well as the prostaglandin PGE2, a marker of acute UV-induced inflammatory response [39]. Additionally, we analyzed the impact of UVB on the known senescence biomarker Lamin B1 [27].

To study this mechanistically, we used UVB, urban dust, and diesel exhaust and measure their impact on keratinocyte SASP-related inflammatory response in monolayer cultures. We found that all these stressors showed induction of inflammation. Since Nam was previously reported to downregulate gene expression of several cytokines, including IL-6, in response to UVB [40], we wished to further explore this effect at the metabolite and protein levels. Nam treatment significantly inhibited the increase of cytokines and prostaglandins synthesized after stress and significantly restored the reduced levels of Lamin B1 (a biomarker known to be lost during senescence) induced by UVB exposure. Relative to cigarette smoke, it has been reported that exposure to keratinocytes can elicit a strong oxidative stress response based on proteomics [41]. We did not detect as strong of a cytokine induction but did see a significant effect of Nam at inhibiting the induced levels of the prostaglandin PGE2. Nam also partially reversed gene expression patterns related to apoptosis, DNA repair, and cell cycle regulation. 
It has been reported that $\mathrm{PM}_{2.5}$ can elicit apoptosis and ROS formation in keratinocytes and the authors showed that this can be prevented by Nam [42]. However, the experiments were performed under culture conditions in which keratinocytes came directly in contact to a suspension of $\mathrm{PM}_{2.5}$. Usage of a 3D skin equivalent model that shows an established barrier and intact stratum corneum would more closely simulate physiological exposure conditions of PM2.5 coming in direct contact to skin with subsequent release of chemical agents bound to the particles [43]. To further simulate daily sun exposure, we also tested the combination of $\mathrm{PM}_{2.5}$ with UVB which showed synergistic induction of inflammation. Interestingly, the complete inhibition by Nam supports that Nam usage may provide a level of protection when skin is exposed to particulate matter during the day when skin is most vulnerable to the synergized stressors.

Steady state oxygen tension in the epidermis of skin is maintained by supplying oxygen from the environment as well as by microcapillary beds residing in the dermis below the basement membrane [44]. While the relative oxygen concentration in human epidermis has been estimated to range between $0.2 \%$ and $8 \%$ [23], epidermal keratinocytes are commonly cultured in vitro under ambient oxygen conditions of $20-22 \%$. These oxygen culture conditions could theoretically be considered "hyperoxic" whereas growing keratinocytes below $8 \%$ would more closely mimic in vivo "normoxic" levels. In studying a potential differential response under high and low oxygen conditions, we found that keratinocytes grown at $5 \% 0_{2}$ had a significantly heightened response to UVB-induced PGE2 synthesis as well as a greater percentage inhibitory effect by Nam. It should be noted that this does not invalidate data generated with keratinocytes grown at ambient oxygen conditions since the relative response to UVB and Nam treatment are similar with the only difference being in the dynamic response range. Part of this oxygen differential phenomenon may be due to greater reliance on glycolysis for basal metabolism and how keratinocytes respond to UV-induced stress [45].

Finally, we show that prior treatment of skin with Nam can help to partially mitigate induction of inflammation by UV exposure via quantitation of the ratio of the skin surface biomarkers IL$1 \alpha \mathrm{RA} / \mathrm{IL}-1 \alpha$. It has been reported that this ratio is directly correlated with inflammation induced by UV radiation [45]. Additionally, IL-1 $\alpha$ is known to be a component of SASP [47]. This measure of reduced inflammation by Nam was supported by a lower quantitated degree of erythema induced by UV. We do not believe that this affect was due to physical or chemical absorption of UV by Nam because there was no product application on the day of UV exposure and Nam does not have strong absorption in the UVA and UVB wavelength region.

In total, this work contributes to the growing fundamental understanding of the impact of environmental stressors on skin biology. Additionally, the data presented here provide further proof to the growing body of evidence that inflammation induced by environmental stressors can be inhibited by Nam to help prevent premature aging and maintain skin's overall homeostasis.

\section{Conclusions}

In this study we show that a SASP-related inflammatory response can be stimulated in skin models when exposed to environmental stressors. Keratinocytes exposed to UVB, urban dust, diesel exhaust, and cigarette smoke extract as well as a skin equivalent models exposed to the combination 
of $\mathrm{PM}_{2.5}$ and $\mathrm{UVB}$, both showed significant increases in quantitated levels of the inflammatory mediators PGE2, IL-6, and IL-8, while UVB also reduced the levels of Lamin B1, known to decrease with senescence. Treatment with Nam was shown to significantly inhibit this stress-induced inflammatory response that is associated with IL-1 $\alpha$ induction. This included testing under more normoxic oxygen culture conditions which magnified Nam inhibition percentage of UVB-induced inflammation. Additionally, we showed that treatment of skin with Nam could partially mitigate the level of IL-1 $\alpha$ related inflammation and erythema elicited by solar simulated radiation. These findings support the hypothesis that Nam could help mitigate premature aging of skin and maintain overall skin homeostasis and health.

Author Contributions: Conceptualization of this work was led by J.E.O., methodology by J.C.B., T.L., B.C.H., T.M., S.B. and C.E.M..; validation by T.M.; formal analysis by T.M. and S.B.; investigation by J.E.O., J.C.B., T.L., B.C.H., T.M., S.B., C.Y.R.T., M.M. and C.E.M.; data curation by J.E.O., J.C.B., T.L., T.M., S.B.; writing-original draft preparation by J.E.O; writing - review and editing by J.E.O., J.C.B., T.L., B.C.H., T.M., C.E.M., S.B.; visualization by J.E.O.; project administration by J.E.O., C.E.M., and S.B.

Funding: This research was supported by The Procter \& Gamble Company and the Agency for Science Technology and Research (A*STAR).

Acknowledgments: We wish to thank Professor Jiang Jingkun of Tsinghua University, Beijing, China for providing PM2.5 samples. We wish to acknowledge Suska Bentz and Amy Altemeier for clinical support and statistical analysis, Jay P. Tiesman and Rachel Adams for providing microarray processing support, Gina M. Fadayel and Ken R. Wehmeyer for providing tape strip protein analysis.

Conflicts of Interest: All authors, except C.Y.R.T. M.M. and S.B., are employees of The Procter \& Gamble Company. All authors declare no conflict of interest.

\section{References}

1. D'Orazio, J., Jarrett, S., Amaro-Ortiz, A., and Scott, T. UV radiation and the skin. Int J Mol Sci. 2013, 14:12222-12248.

2. Yaar, M. and Gilchrest, B.A. Photoaging: mechanism, prevention and therapy. Br J Dermatol. 2007, 157:874887.

3. Mancebo, S.E. and Wang S.Q. Recognizing the impact of ambient air pollution on skin health. J Eur Acad Dermatol Venereol. 2015, 29:2326-2332.

4. Araviiskaia E, Berardesca E, Bieber T, Gontijo G, Sanchez Viera M, Marrot L, Chuberre B, Dreno B. The impact of airborne pollution on skin. J Eur Acad Dermatol Venereol. 2019, 33:1496-1505.

5. Schikowski, T. and Hüls, A. Air pollution and skin aging. Curr Environ Health Rep. 2020, doi: 10.1007/s40572-020-00262-9

6. Valacchi, G., Sticozzi, C., Pecorelli, A., Cervellati, F., Cervellati, C., and Maioli, E. Cutaneous responses to environmental stressors. Ann N Y Acad Sci. 2012, 1271:75-81.

7. Kuehne, A., Emmert, H., Soehle, J., Winnefeld, M., Fischer, F., Wenck, H., Gallinat, S., Terstegen, L., Lucius, R., Hildebrand, J., and Zamboni, N. Acute activation of oxidative pentose phosphate pathway as first-line response to oxidative stress in human skin cells. Mol Cell. 2015, 59:359-371.

8. Giacomoni, P.U. and Rein, G. Factors of skin aging share common mechanisms. Biogerenotology, 2001, 2:219229.

9. Newton, V.L., Mcconnell, J.C., Hibbert, S.A., Graham, H.K., and Watson, R.E. Skin aging: molecular pathology, dermal remodelling and the imaging revolution. G Ital Derm Venereol. 2015, 150:665-674.

10. Bennett, M.F., Robinson, M.K., Baron, E.D., and Cooper, K.D. Skin immune systems and inflammation: protector of the skin or promoter of aging? J Inv Derm Symp Proc. 2008, 13:15-9. 
11. Zhuang, Y. and Lyga, J. Inflammaging in skin and other tissues - the roles of complement system and macrophage. Inflamm Allergy Drug Targets. 2014, 13:153-161.

12. Matts, P.J., Oblong, J.E., and Bissett, D.L. A review of the range of effects of niacinamide in human skin. IFSCC 2002, 50: 285-290.

13. Wohlrab, J. and Kreft, D. Niacinamide - mechanisms of action and its topical use in dermatology. Skin Pharmacol Physiol. 2014, 27:311-315.

14. Surjana, D. and Damian, D.L. Nicotinamide in dermatology and photoprotection. Skinmed. 2011, 9:360-365.

15. Sivapirabu, G., Yiasemides, E., Halliday, G.M., Park, J., and Damian, D.L. Topical nicotinamide modulates cellular energy metabolism and provides broad-spectrum protection against ultraviolet radiation-induced immunosuppression in humans. Br J Derm. 2009, 161:1357-1364.

16. Zhang, X.M., Jing, Y.P., Jia, M.Y., and Zhang, L. Negative transcription regulation of inflammatory genes by group B3 vitamin nicotinamide. Mol Biol Rep, 2012, 39:1036-10371.

17. Snaidr, V.A., Damian, D.L., and Halliday, G.M. Nicotinamide for photoprotection and skin cancer chemoprevention: A review of efficacy and safety. Exp Dermatol, 2019, 28 Suppl 1: 15-22.

18. Ungerstedt, J.S., Blömback, M. and Söderström, T. Nicotinamide is a potent inhibitor of proinflammatory cytokines. Clin Exp Immunol. 2003, 131:48-52.

19. Bhaumik, D., Scott, G.K., Schokrpur, s., Patil, C.K., Orjalo, A.V., Rodier, F., Lithgow, G.J., and Campisi, J. MicroRNAs miR-146a/b Negatively Modulate the Senescence-Associated Inflammatory Mediators IL-6 and IL-8. Aging (Albany NY), 2009, 1:402-411.

20. Campisi, J., Andersen, J.K., Kapahi, P., and Melov, S. Cellular senescence: a link between cancer and agerelated degenerative disease? Semin Cancer Biol. 2011, 21:354-359.

21. Wu, G., Ruben, M.D., Schmidt, R.E., Francey, L.J., Smith, D.F., Anafi, R.C., Hughey, J.J., Tasseff, R., Sherrill, J.D., Oblong, J.E., Mills, K.J., and Hogenesch, J.B. Population-level rhythms in human skin with implications for circadian medicine. Proc Natl Acad Sci U S A. 2018, 115:12313-12318.

22. Tan, C.L., Chin, T., Tan, C.Y.R., Rovito, H.A., Quek, L.S., Oblong, J.E., and Bellanger, S. Nicotinamide Metabolism Modulates the Proliferation/Differentiation Balance and Senescence of Human Primary Keratinocytes. J Invest Dermatol. 2019, 139:1638-1647.

23. Evans, S.M., Schrlau, A.E., Chalian, A.A., Zhang, P., and Koch, C.J. Oxygen levels in normal and previously irradiated human skin as assessed by EF5 binding. J Invest Dermatol. 2006, 126:2596-2606.

24. Su, Y., Han, W., Giraldo, C., De Li, Y., and Block, E.R. Effect of cigarette smoke extract on nitric oxide synthase in pulmonary artery endothelial cells. Am J Cell Mol Biol, 1998, 19:819-825.

25. Langfelder, P. and Horvath, S. Eigengene networks for studying the relationships between co-expression modules. BMC systems biology, 2007, 1.1: 54.

26. Hendrix, S.W., Miller, K.H., Youket, T.E., Adam, R., O'Connor, R.J., Morel, J.G., and Tepper, B.E. Optimization of the skin multiple analyte profile bioanalytical method for determination of skin biomarkers from D-Squame tape samples. Skin Res Technol 2007, 13:330-342.

27. Wang, A.S., Ong, P.F., Chojnowski, A., Clavel, C., and Dreesen, O. Loss of lamin B1 is a biomarker to quantify cellular senescence in photoaged skin. Sci Rep, 2017, 7:15678.

28. Lin, Y., Yang, J., Fu, Q., Ruan, T., and Jiang, G. Exploring the occurrence and temporal variation of ToxCast chemicals in fine particulate matter using suspect screening strategy. Environ Sci Technol. 2019, 53:5687-5696.

29. Robinson MK. Non-invasive clinical assessment of skin irritation/inflammation. In: Barel AO, Paye M, Maibach, H.I., eds. Handbook of Cosmetic Science and Technology, 3rd edn. New York, NY: Informa HealthCare USA, 2009, 481-488.

30. Parrado, C., Mercado-Saenz, S., Perez-Davo, A., Gilaberte, Y., Gonzalez, S., and Juarranz, A. Environmental Stressors on Skin Aging. Mechanistic Insights. Front Pharmacol. 2019, 10:759.

31. Riediker, M., Zink, D., Kreyling, W., Oberdörster, G., Elder, A., Graham, U., Lynch, I., Duschl, A., Ichihara, G., Ichihara, S., Kobayashi, T., Hisanaga, N., Umezawa, M., Cheng, T.J., Handy, R., Gulumian, M., Tinkle, S., and Cassee, F. Particle toxicology and health - where are we? Part Fibre Toxicol. 2019, 16:19.

32. Larrieu, S., Lefranc, A., Gault, G., Chatignoux, E., Couvy, F., Jouves, B., and Filleul, L. Are the short-term effects of air pollution restricted to cardiorespiratory diseases? Am J Epidemiol. 2009, 169:1201-1208.

33. Puri, P., Nandar, S.K., Kathuria, S., and Ramesh, V. Effects of air pollution on the skin: A review. Indian J Dermatol Venereol Leprol. 2017, 83:415-423.

34. Hassoun, Y., James, C., and Bernstein, D.I. The effects of air pollution on the development of atopic disease. Clin Rev Allergy Immunol. 2019, 57:403-414. 
35. Martires KJ, Fu P, Polster AM, Cooper KD, Baron ED. Factors that affect skin aging: a cohort-based survey on twins. Arch Dermatol. 2009, 145:1375-1379.

36. Romani, A., Cervellati, C., Muresan, X.M., Belmonte, G., Pecorelli, A., Cervellati, F., Benedusi, M., Evelson, P., and Valacchi, G. Keratinocytes oxidative damage mechanisms related to airborne particle matter exposure. Mech Ageing Dev. 2018, 86-95.

37. Ryu, Y.S., Kang, K.A., Piao, M.J., Ahn, M.J., Yi, J.M., Hyun, Y.M., Kim, S.H., Ko, M.K., Park, C.O., and Hyun, J.W. Particulate matter induces inflammatory cytokine production via activation of NFkB by TLR5-NOX4ROS signaling in human skin keratinocyte and mouse skin. Redox Biol. 2019, 21:101080.

38. Rea, I.M., Gibson, D.S., McGilligan, V., McNerlan, S.E., Alexander, H.D., and Ross, O.A. Age and AgeRelated Diseases: Role of Inflammation Triggers and Cytokines. Front Immunol. 2018, 9:586.

39. Rhodes, L.E., Gledhill, K., Masoodi, M., Haylett, A.K., Brownrigg, M., Thody, A.J., Tobin, D.J., and Nicolaou, A. The sunburn response in human skin is characterized by sequential eicosanoid profiles that may mediate its early and late phases. FASEB J. 2009, 23:3947-3956.

40. Monfrecola G, Gaudiello F, Cirillo T, Fabbrocini G, Balato A, Lembo S. Nicotinamide downregulates gene expression of interleukin-6, interleukin-10, monocyte chemoattractant protein-1, and tumour necrosis factor- $\alpha$ gene expression in HaCaT keratinocytes after ultraviolet B irradiation. Clin Exp Dermatol. 2013, 38:185-188.

41. Rajagopalan, P., Nanjappa, V., Raja, R., Jain, A.P., Mangalaparthi, K.K., Sathe, G.J., Babu, N., Patel, K., Cavusoglu, N., Soeur, J., Pandey, A., Roy, N., Breton, L., Chatterjee, A., Misra, N., and Gowda, H. How Does Chronic Cigarette Smoke Exposure Affect Human Skin? A Global Proteomics Study in Primary Human Keratinocytes. OMICS. 2016, 20:615-626.

42. Zhen, A.X., Piao, M.J., Kang, K.A., Fernando, P.D.S.M., Kang, H.K., Koh, Y.S., Yi, J.M., and Hyun, J.W. Niacinamide protects skin cells from oxidative stress induced by particular matter. Biomol Ther, 2019, 5:562569.

43. Sah, D., Verma, P.K., Kumari, K.M., and Lakhani, A. Chemical partitioning of fine particle-bound As, Cd, $\mathrm{Cr}, \mathrm{Ni}, \mathrm{Co}, \mathrm{Pb}$ and assessment of associated cancer risk due to inhalation, ingestion and dermal exposure. Inhal Toxicol. 2017, 29:483-493.

44. Stücker, M., Struk, A., Altmeyer, P., Herde, M., Baumgärtl, H., and Lübbers, D.W. The cutaneous uptake of atmospheric oxygen contributes significantly to the oxygen supply of human dermis and epidermis. $J$ Physiol. 2002, 538:985-994.

45. Park J, Halliday GM, Surjana D, Damian DL. Nicotinamide prevents ultraviolet radiation-induced cellular energy loss. Photochem Photobiol. 2010, 86:942-948.

46. Hirao, T., Aoki, H., Yoshida, T., Sato, Y., and Kamoda, H. Elevation of interleukin 1 receptor antagonist in the stratum corneum of sun-exposed and ultraviolet B-irradiated human skin. J Invest Dermatol. 1996, 106:1102-1107.

47. Wiley, C.D., Velarde, M.C., Lecot, P., Liu, S., Sarnoski, E.A., Freund, A., Shirakawa, K., Lim, H.W., Davis, S.S., Ramanathan, A., Gerencser, A.A., Verdin, E., and Campisi, J. Mitochondrial Dysfunction Induces Senescence with a Distinct Secretory Phenotype. Cell Metab. 2016, 23:303-314. 